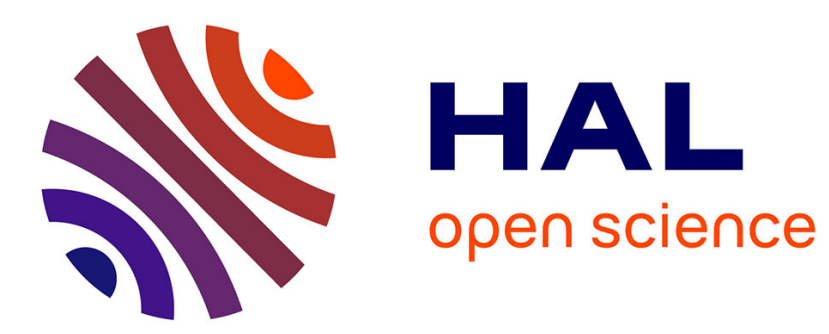

\title{
Nonlinear Doppler reflectometry power response. Analytical predictions and full-wave modelling
}

\author{
O L Krutkin, E. Gusakov, Stéphane Heuraux, C Lechte
}

\section{To cite this version:}

O L Krutkin, E. Gusakov, Stéphane Heuraux, C Lechte. Nonlinear Doppler reflectometry power response. Analytical predictions and full-wave modelling. Plasma Physics and Controlled Fusion, 2019, 61 (4), pp.045010. 10.1088/1361-6587/ab0236 . hal-02434260

\section{HAL Id: hal-02434260 \\ https://hal.univ-lorraine.fr/hal-02434260}

Submitted on 9 Jan 2020

HAL is a multi-disciplinary open access archive for the deposit and dissemination of scientific research documents, whether they are published or not. The documents may come from teaching and research institutions in France or abroad, or from public or private research centers.
L'archive ouverte pluridisciplinaire HAL, est destinée au dépôt et à la diffusion de documents scientifiques de niveau recherche, publiés ou non, émanant des établissements d'enseignement et de recherche français ou étrangers, des laboratoires publics ou privés. 


\title{
Nonlinear Doppler reflectometry power response. Analytical predictions and full-wave modelling.
}

\author{
O L Krutkin ${ }^{1,2}$, E Z Gusakov ${ }^{1}$, S Heuraux ${ }^{2}$ and C Lechte ${ }^{3}$ \\ ${ }^{1}$ Ioffe Institute, St. Petersburg, Russia \\ ${ }^{2}$ Institute Jean Lamour UMR 7198 CNRS, Université de Lorraine, 54000 Nancy, France \\ ${ }^{3}$ Institute of Interfacial Process Eng. and Plasma Technology, 70569 Stuttgart, Germany \\ E-mail: krutkinoleg@rambler.ru
}

Received xxxxxx

Accepted for publication $\mathrm{xxxxx}$

Published xxxxxx

\section{Abstract}

The transition of Doppler reflectometry diagnostic to nonlinear regime of scattering is studied analytically with the use of the perturbation theory applied to Helmholtz equation. Density fluctuations with large radial correlation length, O-polarization of the probing beam, linear background density profile and slab geometry are considered. Formulae for onset of nonlinearity are derived for different parameter ranges. Obtained results are generalized for the arbitrary turbulence radial correlation length. The results of physical optics model are reproduced, while new formulae are obtained for different parameter ranges and their experimental relevance is demonstrated. Qualitative explanation for scattered signal nonlinear power dependence is proposed. Analytical results are validated with IPF-FD3D code full-wave numerical modelling for radially uniform and fully 2D turbulence..

Keywords: turbulence diagnostics, Doppler reflectometry, plasma

\section{Introduction}

Turbulent transport plays a key role in limiting confinement in magnetic fusion devices. Investigation of underlying physical processes behind it requires suitable tools to study plasma turbulence.

One of such tools is Doppler reflectometry or Doppler Backscattering based on the analysis of time series obtained at fixed frequencies, which allows to study poloidal spectrum of electron density fluctuations [1] as well as poloidal rotation velocity [2]. The diagnostic probes the plasma with microwave beam in the presence of a turning point. This probing beam is tilted with respect to magnetic surface and only the backscattering signal is measured, not the direct reflection. The difference in frequency between scattering and probing signals is interpreted as a Doppler effect allowing to define the poloidal velocity of density fluctuations. This velocity is further decomposed into plasma rotation velocity and phase velocity of plasma density fluctuations. The tilting angle of antenna in these measurements corresponds to fluctuation poloidal wavenumber defined by Bragg rule, which makes the measurement of fluctuation poloidal wavenumber spectrum possible by varying the probing angle and the probing frequency assuming a poloidal invariance of the turbulence properties.

However, such a straightforward interpretation is only possible in linear regime of scattering, where Born approximation over the amplitude of density fluctuations is applicable. This case was extensively studied both analytically [3] and numerically [4], [5].

Analytical predictions have also been made for strongly nonlinear (or saturated) case [6] and nonlinear effects were observed in full-wave numerical modeling [7]-[9]. In the analytical theory a nonlinearity criterion was derived, that would allow to distinguish strongly nonlinear regime of Doppler reflectometry. 
Finally, a study of intermediate nonlinear regime, when the amplitude of scattered signal scales nonlinearly with density fluctuations r.m.s. was performed [10] and criterion of transition from linear regime to nonlinear regime was derived. This analysis, however, was done in the physical optics approximation, which has limited domain of validity due to the fact that only plasma at turning point region is taken into consideration.

In the present paper, intermediate nonlinear regime of Doppler reflectometry will be considered in the framework of perturbation theory applied to Helmholtz equation, to overcome limitations of physical optics.

\section{Analytical treatment}

In this work, plasma probing with the microwave beam of O-polarization will be considered. Analysis is perfomed in a slab geometry, background density profile is assumed to be linear over radial coordinate and uniform over poloidal one. Model geometry of this treatment is shown on the figure 1.

Like in [10], the transition to nonlinear scattering regime is assumed to happen when amplitude of scattered signal corresponding to linear scattering becomes smaller than that of the signal corresponding to multiple scattering. To determine these signals perturbation theory is used.

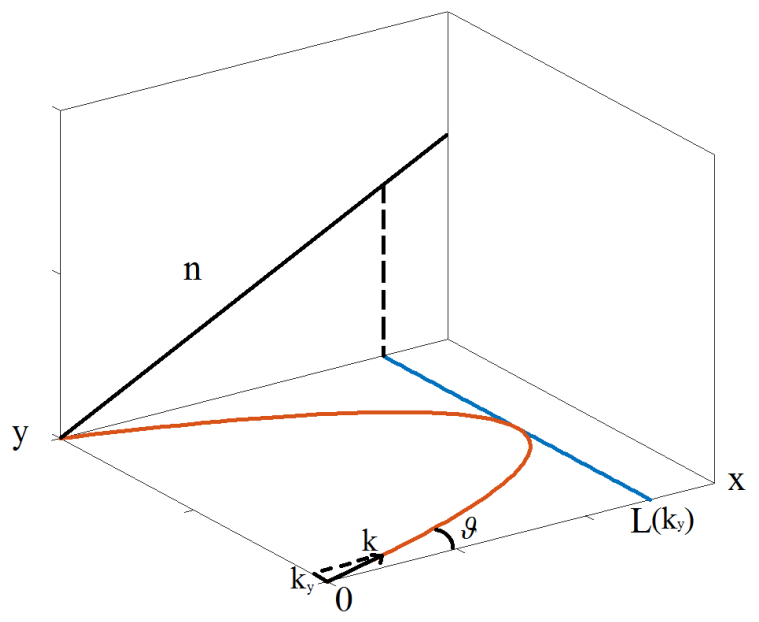

Figure 1. The scheme of geometry used in model. Background density profile is linear along $\mathrm{x}$ direction.

\subsection{Basic equations and perturbation theory}

Microwave propagating through plasma at fixed frequency is described by Helmholtz equation:

$$
\frac{d^{2}}{d x^{2}} E(x, y)+\frac{d^{2}}{d y^{2}} E(x, y)+k(x, y)^{2} E(x, y)=0 ;
$$

which in the case of perturbed plasma can be written as:

$$
\Delta E(x, y)+k(x)^{2} E(x, y)-\delta k(x, y)^{2} E(x, y)=0 ;
$$

The wave number for O-mode is defined as $k^{2}(x, y)=1$ $n(x, y) / n_{c}$ where $n_{c}=m_{e} \omega^{2} / 4 \pi e^{2}$ is cut-off density. That in turn allows us to relate wave number perturbation to density perturbation: $k^{2}(x)=1-n_{0}(x) / n_{c} ; \delta k^{2}(x, y)=\delta n(x, y) / n_{c}$ While for the linear density profile exact solutions can be obtained, we will use WKB approximation. This allows us to obtain all the relevant physical results without dealing with complicated mathematical expressions. Assuming $\delta k=0$ in equation to (2) and separating variables, WKB approximation, solution of unperturbed Helmholtz equation for a poloidal harmonic $k_{y}$ is:

$$
\begin{aligned}
& E^{0}\left(x, k_{y}\right)=f\left(k_{y}\right) \sqrt{\frac{k\left(0, k_{y}\right)}{k\left(x, k_{y}\right)}} \times \\
& \times \mathrm{e}^{i \Phi\left(k_{y}\right)+i \frac{\pi}{4}} \cos \left(\int_{x}^{L\left(k_{y}\right)} k\left(x, k_{y}\right) d x+\frac{\pi}{4}\right) ; \\
& L\left(k_{y}\right)
\end{aligned}
$$

where $\Phi\left(k_{y}\right)=\int_{0}^{L} k\left(x, k_{y}\right) d x, f\left(k_{y}\right)$ is the probing beam amplitude distribution over poloidal wavenumbers. $L\left(k_{y}\right)$ is radial turning point position for a given poloidal wave number and $k\left(x, k_{y}\right)^{2}=k^{2}(x)-k_{y}^{2}$.

Equation describing the next order of perturbation theory for a single poloidal mode can be written as:

$$
\begin{aligned}
& \frac{d^{2}}{d x^{2}} \frac{c^{2}}{\omega^{2}} E^{1}\left(x, k_{y}\right)+\frac{c^{2}}{\omega^{2}} k\left(x, k_{y}\right)^{2} E^{1}\left(x, k_{y}\right)= \\
& =\sqrt{\frac{k\left(0, k_{y}\right)}{k\left(x, k_{y}\right)}} \int_{-\infty}^{\infty} \frac{\delta n(x, q)}{n_{c}} \exp \left(i \Phi\left(k_{y}-q\right)+i \frac{\pi}{4}\right) \times \\
& \times \cos \left(\int_{x}^{L\left(k_{y}-q\right)} k\left(x^{\prime}, k_{y}-q\right) d x^{\prime}+\frac{\pi}{4}\right) f\left(k_{y}-q\right) d q ;
\end{aligned}
$$

Where $q$ corresponds to the poloidal wave number of density perturbation over which integration is carried out. For further analysis, we will assume that density fluctuations are uniform over $x$, which corresponds to large $\left(l_{c x} \sim L(0)\right)$ radial correlation length and is in line with physical optics description. In this case, the solution to this equation with boundary conditions defined by perturbation theory can be written as:

$$
E^{1}=\sum_{i= \pm 1} \frac{\frac{\omega^{2}}{c^{2}} \frac{\delta n(q)}{n_{c}} f\left(k_{y}-q\right) e^{i(\mathrm{t}+1) \frac{\pi}{4}}}{k\left(x, k_{y}\right)^{2}-k\left(x, k_{y}-q\right)^{2}} \sqrt{\frac{k\left(0, k_{y}-q\right)}{k\left(x, k_{y}-q\right)}} \times
$$

$\times \exp \left(i \Phi\left(k_{y}-q\right)+i t\left(\int_{x}^{L\left(k_{y}-q\right)} k\left(x^{\prime}, k_{y}-q\right) d x^{\prime}\right)\right)-$

$-\sum_{s= \pm 1} \sum_{i= \pm 1} \frac{1}{2}\left(1-t \frac{k\left(0, k_{y}-q\right)}{k\left(0, k_{y}\right)}\right) \frac{\frac{\omega^{2}}{c^{2}} \frac{\delta n(q)}{n_{c}} f\left(k_{y}-q\right) e^{i(s+1) \frac{\pi}{4}}}{k\left(0, k_{y}\right)^{2}-k\left(0, k_{y}-q\right)^{2}} \times$

$\times \sqrt{\frac{k\left(0, k_{y}\right)}{k\left(x, k_{y}\right)}} \exp \left(i \Phi\left(k_{y}\right)+(t+1) \Phi\left(k_{y}\right)+i s\left(\int_{x}^{L\left(k_{y}\right)} k\left(x^{\prime}, k_{y}\right) d x^{\prime}\right)\right)$; 
The solution (5) includes two terms - one of them represents a solution of the differential equation (4) when it is homogeneous, whereas the other does not. The latter term can be interpreted as a "forced" oscillation of system, while the former is the propagating one. For this reason, we will designate this terms as $A^{F}$ and $A^{P}$.

$$
\begin{aligned}
& A^{F}=\sum_{p= \pm 1} \sqrt{\frac{k(0, K)}{k(x, K)}} \exp \left(i \Phi(K)+i p\left(\int_{x}^{L(K)} k\left(x^{\prime}, K\right) d x^{\prime}+\frac{\pi}{4}\right)\right) ; \\
& A^{P}=\sum_{p= \pm 1} \sum_{t= \pm 1} \frac{1}{2}\left(1-\frac{t k(0, K)}{k(0, K+q)}\right) \sqrt{\frac{k(0, K+q)}{k(x, K+q)}} \times \exp \left(i p \frac{\pi}{4}\right) \\
& \left.\times \exp \left(i \Phi(K+q)+i(t+1) \Phi(K)+i p \int_{x}^{L(K+q)} k\left(x^{\prime}, K+q\right) d x^{\prime}\right)\right]
\end{aligned}
$$

Now, instead of deriving the solution for the next order of perturbation theory it is easier to use reciprocity theorem [11]

$$
A_{s}=\frac{i e^{2}}{4 m_{e} \omega^{2}} \omega \sqrt{P} \int_{-\infty}^{\infty} \int_{-\infty}^{\infty} \frac{\delta n(y)}{n_{c}} E(x, y)^{2} d x d y ;
$$

Here $A_{s}$ is the amplitude of scattered signal received by antenna and $P$ is probing beam power. This formula allows us to derive the signals corresponding to linear and quadratic scattering without calculating corresponding electric field in the whole space.

\subsection{Linear response}

To calculate the scattering signal corresponding to a single scattering, electric field given by formula (3) should be substituted into (6).

$A_{s}=-\frac{e^{2} \sqrt{P}}{4 m_{e} \omega} \int_{-\infty}^{\infty} d q \int_{-\infty}^{\infty} d k_{y} \int_{0}^{\min \left(L\left(k_{y}\right), L\left(k_{y}+q\right)\right)} d x \sum_{s, t= \pm 1} \frac{\delta n(q)}{n_{c}} \mathrm{e}^{i(s+t) \frac{\pi}{4}} \times$

$f\left(-k_{y}-q\right) \sqrt{\frac{k\left(0, k_{y}\right) k\left(0, k_{y}+q\right)}{k\left(x, k_{y}\right) k\left(x, k_{y}+q\right)}} \exp \left(i \Phi\left(k_{y}+q\right)+i \Phi\left(k_{y}\right)\right) \times$

$\times f\left(k_{y}\right) \exp \left(i t \int_{x}^{L\left(k_{y}\right)} k\left(x^{\prime}, k_{y}\right) d x^{\prime}+i s \int_{x}^{L\left(k_{y}+q\right)} k\left(x^{\prime}, k_{y}+q\right) d x^{\prime}\right)$;

We will consider the version of diagnostic where the same antenna used as receiver and emitter. Also the diagram of antenna will be assumed Gaussian so that:

$$
f\left(k_{y}\right)=(2 \sqrt{\pi} \rho)^{1 / 2} \exp \left[-\left(k_{y}-K\right)^{2} \rho^{2} / 2\right] ;
$$

where $K=\omega \sin \vartheta / c, \vartheta$ is a probing angle with respect to normal to magnetic surface (which in our case is directed along $\mathrm{x}$ ) and $2 \rho$ is probing beam waist. The integrals over $q$ and $k_{y}$ in (7) can be calculated using saddle-point method, however to simplify further derivation we will assume wide enough beam corresponding to the cutoff situated in the antenna near field.

$\rho^{2}>>\int_{0}^{L(K)} \frac{d x}{k(x, K)}$
In this case antenna diagram determines the main contribution to integrals over poloidal wave numbers in (7) and the result of integration is

$$
\begin{aligned}
& A_{s}=\frac{i e^{2}}{4 m_{e} \omega^{2}} \frac{4 \pi^{3 / 2}}{\rho} \omega \sqrt{P} \int_{0}^{L(K)} d x \sum_{t= \pm 1} \sum_{s= \pm 1} \frac{\delta n(-2 K)}{n_{c}} \frac{k\left(0, k_{y}\right)}{k(x, K)} \times \\
& \times \exp \left(2 i \Phi(K)+i \frac{\pi}{2}+i(s+t)\left[\int_{x}^{L\left(k_{y}\right)} k(x, K) d x+\frac{\pi}{4}\right]\right) ;
\end{aligned}
$$

In the formula (9) two contributions of different origin can be seen. Contributions possessing same indexes $\mathrm{s}=\mathrm{t}$, which correspond to backscattering in radial direction and contributions possessing $\mathrm{s}=-\mathrm{t}$ which correspond to the forward-scattering.

Up to this point, we didn't make any assumptions about density profile shape. To calculate integral over $\mathrm{x}$ we will assume that density profile is linear, which means that $\mathrm{k}(\mathrm{x})$ can be written as $k\left(x, k_{y}\right)=\frac{\omega}{c} \sqrt{\frac{L\left(k_{y}\right)-\mathrm{x}}{L(0)}}$

Which allows us to calculate the scattering signal, which for large radial correlation length $\left(l_{c x}>>\left(L c^{2} / \omega^{2}\right)^{1 / 3}\right)$ is dominated by forward-scattering.

$$
\begin{aligned}
& A_{s}^{l i n}=-4 \frac{\pi^{3 / 2} e^{2} \sqrt{P}}{m_{e} \omega \rho} \frac{\delta n(-2 K)}{n_{c}} L(K) \times \\
& \times \exp \left(i \frac{4}{3} \frac{\omega L(K)^{3 / 2}}{c L(0)^{1 / 2}}\right)
\end{aligned}
$$

\subsection{Quadratic response}

Next, we calculate the quadratic response to the backscattering signal by substituting formulae (3) and (5) into (6). In the case of wide enough antenna beam (8) that we are considering, this result in an expression:

$$
\begin{aligned}
& A_{s}=\frac{e^{2}}{4 m_{e} \omega} \frac{4 \pi^{3 / 2}}{\rho} \sqrt{P} \frac{\omega^{2}}{c^{2}} \int_{-\infty}^{\infty} d q \int_{0}^{L_{\min }} d x \sum_{s= \pm 1} \frac{\delta n(q)}{n_{c}} \frac{\delta n(-q-2 K)}{n_{c}} \times \\
& \exp \left(i \Phi(K)+i s \int_{x}^{L(K)} k(x, K) d x+i s \frac{\pi}{4}\right) \sqrt{\frac{k(0, K)}{k(x, K)} \frac{\left(A^{F}-A^{N}\right)}{q(q+2 K)}}
\end{aligned}
$$

Here $L_{\text {min }}$ is the smallest of $L(q)$ and $L(q+K)$.

Firstly, we will consider $A^{F}$ together with the $t=-1$ term of $A^{P}$ since they can cancel each other out for specific values of $q$. Integrals over $\mathrm{x}$ can be written as:

$I_{F}=\sum_{s, p= \pm 1} \int_{0}^{L(K)} \frac{k(0, K)}{k(x, K)} \exp \left(i \Phi(K)+i(s+p)\left[\int_{x}^{L(K)} k\left(x^{\prime}, K\right) d x^{\prime}+\frac{\pi}{4}\right]\right) d x ;$

$I_{P}=\sum_{s, p= \pm 1} \int_{0}^{L_{\text {min }}} \sqrt{\frac{k(0, K)}{k(x, K)} \frac{k(0, K+q)}{k(x, K+q)}} \exp (i \Phi(K+q)) \times$

$\times \exp \left(i s\left[\int_{x}^{L(K)} k\left(x^{\prime}, K\right) d x^{\prime}+\frac{\pi}{4}\right]+i p\left[\int_{x}^{L(K+q)} k\left(x^{\prime}, K+q\right) d x^{\prime}+\frac{\pi}{4}\right]\right) d x ;$

Firstly, the main contribution to the signal is once again provided by forward-scattering $(s=p)$ due to faster variation of phase in the backscattering term integrand. $I_{P}$ coincides with $I_{F}$ only for $q=0,-2 K$, and is smaller for other values of $q$, also due to variation of phase of the 
integrand. For that reason we will consider the vicinity of $q=0$ where phase of integrand in $I_{P}$ can be expanded into Taylor series. Since the integrand in (12) is symmetrical around $q=-K$, to calculate the integral over $\mathrm{q}$ it is enough to consider $q>-K$. That area is defined by condition $|q| \ll \Delta q=\frac{1}{\sqrt{L(0) L(K)} \operatorname{sin\vartheta }}$, which corresponds to slow variation of integrand phase over integration domain. This consideration however is only valid, when $q=0$ and $q=-2 K$ can be considered separately, which corresponds to $K>\Delta q$.

1.3.1 Large probing angle $K>\Delta q$.

After performing the integration over $x$ under the same assumptions as in 1.2 we obtain the following:

$$
\begin{aligned}
& I_{F}^{F S}=4 L(K) \exp \left(i \frac{2}{3} \frac{\omega L(K)^{3 / 2}}{c L(0)^{1 / 2}}\right) ; \\
& I_{P}^{F S}=4 L(K) \exp \left(i \frac{2}{3} \frac{\omega L(K)^{3 / 2}}{c L(0)^{1 / 2}}-2 i \sqrt{L(0) L(K)} \frac{c}{\omega} K q\right) \times \\
& \times \frac{\sin \left(2 \sqrt{L(0) L(K)} \frac{c}{\omega} K q\right)}{2 \sqrt{L(0) L(K)} \frac{c}{\omega} K q} ;
\end{aligned}
$$

A similar result can be obtained for $t=1$ term in $A^{P}$. It is however, smaller than a combination of terms in (13) and can be neglected. Due to symmetry of integrand, same results can be obtained in vicinity of $q=-2 K$.

Substituting (13) into (11) and neglecting $A^{P}$ where it is appropriate we get

$$
\begin{aligned}
& A_{s}=2 \frac{e^{2}}{4 m_{e} \omega^{2}} \frac{4 \pi^{3 / 2}}{\rho} \omega \sqrt{P} \frac{\omega^{2}}{c^{2}} 4 L(K) \exp \left(i \frac{4}{3} \frac{\omega L(K)^{3 / 2}}{c L(0)^{1 / 2}}\right) \times \\
& \times\left[\int_{-K}^{-\Delta q} \frac{\delta n(q) \delta n(-q-2 K)}{q(q+2 K) n_{c}{ }^{2}} d q+\int_{\Delta q}^{\infty} \frac{\delta n(q) \delta n(-q-2 K)}{q(q+2 K) n_{c}{ }^{2}} d q+\right. \\
& \left.+i \sqrt{L(0) L(K)} \frac{c}{\omega} \int_{-\Delta q}^{\Delta q} \frac{\delta n(q) \delta n(-q-2 K)}{n_{c}{ }^{2}} d q\right]
\end{aligned}
$$

To continue analysis, we will assume the Gaussian spectrum of the density fluctuations $\delta n(q)=\delta n_{0} l_{c y} \exp \left[-q^{2} l_{c y}^{2} / 8\right]$. In this case, estimating the main contribution to $\mathrm{A}_{\mathrm{s}}$ one can obtain:

$$
\left\{\begin{array}{l}
A_{s} \propto \frac{e^{2} \sqrt{P}}{m_{e} \omega \rho} \frac{\omega^{2}}{c^{2}} \frac{L(K) l_{c y}}{K^{2}} \frac{\delta n_{0}{ }^{2}}{n_{c}} \times \\
\times \exp \left(i \frac{4}{3} \frac{\omega L(K)^{3 / 2}}{c L(0)^{1 / 2}}-\frac{l_{c y}^{2} K^{2}}{4}\right) ; \\
A_{s} \propto \frac{e^{2} \sqrt{P}}{m_{e} \omega \rho} \frac{\omega^{2}}{c^{2}} \frac{L(K) l_{c y}^{2}}{K} \frac{\delta n_{0}^{2}}{n_{c}{ }^{2}} \times 1 ; \quad K l_{c y}<1 ; \\
\times \exp \left(i \frac{4}{3} \frac{\omega L(K)^{3 / 2}}{c L(0)^{1 / 2}}\right) ;
\end{array}\right.
$$

In the intermediate case $K l_{c y} \sim 1$ estimating integral is difficult, but these two parametric dependencies coincide with each other in this case which points to the conclusion that parametric dependencies properly describe the whole parameter interval.

\subsubsection{Small probing angle $K<\Delta q$.}

As in the previous case, we expand the integrand phase in (12) in the vicinity of $q=0$ in Taylor series. This time the area of validity of such expansion is $q \ll \Delta q_{2}=\sqrt{\omega / c L(0) \cos \vartheta} . \quad$ After performing integration over $\mathrm{x}$ under the same assumptions as before we get

$I_{F}^{F S}=4 L(K) \exp \left(i \frac{2}{3} \frac{\omega L(K)^{3 / 2}}{c L(0)^{1 / 2}}\right)$

$I_{P}^{F S} \approx 4 L(K) \exp \left(i \frac{2}{3} \frac{\omega L(K)^{3 / 2}}{c L(0)^{1 / 2}}-i \sqrt{L(K) L(0)} \frac{c}{\omega} q(q+K)\right) \times$

$\times \frac{\sin \left(\sqrt{L(K) L(0)} \frac{c}{\omega} q(q+K)\right)}{\sqrt{L(K) L(0)} \frac{c}{\omega} q(q+K)} ;$

Once again, taking the contribution of $A^{P}$ into account only in vicinity of $q=0$ and $q=-2 K$, we can divide $A_{s}$ into two integrals over different domains:

$$
\begin{aligned}
& A_{s}=8 \frac{e^{2}}{m_{e} \omega} \frac{\pi^{3 / 2}}{\rho} \sqrt{P} \frac{\omega^{2}}{c^{2}} L(\mathrm{~K}) \exp \left(i \frac{2}{3} \frac{\omega L(K)^{3 / 2}}{c L(0)^{1 / 2}}\right) \times \\
& \times\left[\int_{\Delta q_{2}}^{\infty} \frac{\delta n(q) \delta n(-q-2 K)}{q(q+2 K) n_{c}{ }^{2}} d q+\right. \\
& \left.+i \sqrt{L(K) L(0)} \frac{c}{\omega} \int_{-K}^{\Delta q_{2}} \frac{q+K}{q+2 K} \frac{\delta n(q) \delta n(-q-2 K)}{n_{c}{ }^{2}} d q\right]
\end{aligned}
$$

Estimating the main contributions into integral over $q$ for different values of $l_{c y}$ we obtain

$$
\begin{aligned}
& A_{s} \propto \frac{e^{2} \sqrt{P}}{m_{e} \omega \rho} \frac{\delta n_{0}{ }^{2}}{n_{c}{ }^{2}} l_{c y} \sqrt{L(K)^{3} L(0)} \times \\
& \times \frac{\omega}{c} \exp \left(i \frac{4}{3} \frac{\omega L(K)^{3 / 2}}{c L(0)^{1 / 2}}-K^{2} l_{c y}^{2} / 4\right) ; \\
& \Delta q l_{c y}>>1 ; \\
& A_{s} \propto \frac{e^{2} \sqrt{P}}{m_{e} \omega \rho} \frac{\delta n_{0}{ }^{2}}{n_{c}{ }^{2}} l_{c y}^{2} L(K) \frac{\omega^{2}}{c^{2}} \times \\
& \times \sqrt{\frac{\sqrt{L(K) L(0) c}}{\omega}} \exp \left(i \frac{4}{3} \frac{\omega L(K)^{3 / 2}}{c L(0)^{1 / 2}}\right) ; \\
& \Delta q l_{c y} \ll 1 ;
\end{aligned}
$$

These parametric dependencies also coincide in the intermediate parameter range at $\Delta q l_{c y} \sim 1$. Moreover at $K \sim \Delta q$ formulae (15) and (18) are in agreement with each other.

1.4 Criterion for transition to nonlinear backscattering regime 
We have obtained both linear and quadratic contributions to the scattering signal, which correspond to single and double scattering of the probing wave. Comparing these two contributions allows us to derive the threshold amplitude for $\delta n$ exceeding which lead to nonlinear effects dominating in the scattering signal.

Combining (10) with (15) and (18) we obtain following criteria

$\left\{\begin{array}{lll}\frac{\delta n}{n_{c}}>>\frac{c}{\omega L \cos \vartheta} \mathrm{e}^{-K^{2} l_{c y}^{2} / 4} ; & \Delta q_{2} l_{c y}>1 \quad & (a) \\ \frac{\delta n}{n_{c}}>>\frac{c}{\omega l_{c y}} \sqrt{\frac{c}{\omega L \cos \vartheta}} ; & \Delta q_{2} l_{c y}<<1 & (b)\end{array}\right\} \quad \Delta q>>K$

While this system is consistent in itself, interpreting different parameter ranges using it is difficult. This system of inequalities can be rewritten with respect to the values of $L, l_{c y}, c / \omega$ and $\vartheta$. Doing that, we obtain two cases corresponding to different value of poloidal correlation length of turbulence.

For $l_{c y}^{2}>>L c / \omega$ :

$\frac{\delta n}{n_{c}} \gg \frac{c}{\omega L \cos \vartheta} \exp \left(\frac{-K^{2} l_{c y}^{2}}{4}\right) ; \quad \sin \vartheta<\sqrt{\frac{c}{\omega L \cos \vartheta}} ;$
$\frac{\delta n}{n_{c}} \gg>\sin ^{2} \vartheta \exp \left(\frac{-K^{2} l_{c y}^{2}}{4}\right) ; \quad \sqrt{\frac{c}{\omega L \cos \vartheta}} \ll \sin \vartheta$

In the opposite case of ${1_{\mathrm{cy}}}^{2}<<\mathrm{Lc} / \omega$ :

$\frac{\delta n}{n_{c}}>>\frac{c}{\omega l_{c y}} \sqrt{\frac{c}{\omega L \cos \vartheta}} ; \quad \sin \vartheta<<\sqrt{\frac{c}{\omega L \cos \vartheta}} ;$

$\frac{\delta n}{n_{c}}>>\frac{c \sin \vartheta}{\omega l_{c y}}$ $\sqrt{\frac{c}{\omega L \cos \vartheta}} \ll \sin \vartheta$ $\sin \vartheta<\frac{c}{\omega l_{c y}}$

$\frac{\delta n}{n_{c}}>>\sin ^{2} \vartheta \exp \left(\frac{-K^{2} l_{c y}^{2}}{4}\right) ; \quad \frac{c}{\omega l_{c y}} \ll \sin \vartheta ;$

The latter case (21) seems to be relevant to the Doppler backscattering experimental measurements, however for these results to be applicable the density perturbation should be smaller than that prescribed by the criteria of transition to fully nonlinear regime. This regime is characterized by the strong probing wave phase modulation and by saturation of the scattering signal power growth with the probing microwave power. Using the approach described in [12] together with assumptions of linear profile and large radial correlation length the condition of strong phase perturbation of the probing beam can be written in accordance with appendix A as:

$$
\begin{array}{lll}
\frac{\delta n}{n_{c}}>>\frac{c}{\omega L \cos \vartheta} ; & \sin \vartheta<<\frac{l_{c y}}{L \cos \vartheta} ; \quad(a) \\
\frac{\delta n}{n_{c}}>>\frac{c}{\omega} \sqrt{\frac{\sin \vartheta}{l_{c y} L \cos \vartheta}} ; & \sin \vartheta>>\frac{l_{c y}}{L \cos \vartheta} ; \quad(b)
\end{array}
$$

Comparing (22) to (20) and (21) we can see that even for a small nonzero probing angle large enough value of $l_{c y}$ can lead to an existence of nonlinear regime that is not saturated. It can also be seen that in case of $l_{c y}<c / \omega$ the strong phase modulation threshold is never higher than the threshold of nonlinear regime we are considering in this paper.

To draw comparison between obtained criteria and predictions of the physical optics model [10] we first need to change their normalization based on [12][13] allowing to take the plasma volume phenomenon into account. In the case of 1D turbulence considered in the present paper the condition of strong phase modulation utilized for normalisation in [10] instead of $\frac{\delta n}{n_{c}}>>\frac{c}{\omega \sqrt{l_{c x} L \ln \left(L / l_{c x}\right)}}$ takes a form (22). With that the condition of nonlinear regime onset derived in [10] coincides with (20). According to [10], this criterion should be valid for the whole parameter range and other criteria corresponding to (20) are not obtained. The reason for that might be Kirchhoff approximation [14] used in [10]. Under our assumption, the Kirchhoff aproximation imposes condition $\delta n / n_{c}<<c / \omega L$ which contradicts most of parameter ranges in (20) and (21)

Finally, in order to generalise criteria (20) and (21) to the case of real 2D turbulence we will employ the same approach as described in [10]. Firstly, we will note that in the case of 1D density fluctuations and normal probing of plasma the criterion (20) coincides with the saturation criterion (22). While we are unable to derive nonlinearity criterion that would account for radial inhomogenity of the turbulence and include redial correlation length, a saturation criterion at normal incidence of the probing wave was obtained in [[13]. Assuming that the two criteria coincide in general case of the 2D turbulence and using the strong phase modulation criterion $\frac{\delta n}{n_{c}}>>\frac{c}{\omega \sqrt{l_{c x} L \ln \left(L / l_{c x}\right)}}$ obtained for the 2D turbulence in [12] and [13] one can obtain from (20) the following criteria:

$$
\begin{aligned}
& \frac{\delta n}{n_{c}}>>\frac{c \mathrm{e}^{\frac{-K^{2} l_{c y}^{2}}{4}}}{\omega \sqrt{l_{c x} L \ln \left(\frac{L}{l_{c x}}\right) \cos \vartheta}} ; \quad \sin \vartheta<<\sqrt{\frac{c}{\omega L \cos \vartheta}} \\
& \frac{\delta n}{n_{c}} \gg>\sin ^{2} \vartheta \sqrt{\frac{L}{l_{c x} \ln \left(\frac{L}{l_{c x}}\right)}} \mathrm{e}^{\frac{-K^{2} l_{c y}^{2}}{4}} ; \sqrt{\frac{c}{\omega L \cos \vartheta}} \ll \sin \vartheta
\end{aligned}
$$


Taking into account consistency between (20) and (21), a version of latter generalized for 2D fluctuations can be written as:

$$
\begin{aligned}
& \frac{\delta n}{n_{c}}>>\frac{c}{\omega l_{c y}} \sqrt{\frac{c}{\omega l_{c x} \ln \left(\frac{L}{l_{c x}}\right) \cos \vartheta}} ; \quad \sin \vartheta<<\sqrt{\frac{c}{\omega L \cos \vartheta}} ; \\
& \frac{\delta n}{n_{c}}>>\frac{c \sin \vartheta}{\omega l_{c y}} \sqrt{\frac{L}{l_{c x} \ln \left(\frac{L}{l_{c x}}\right)} ;} \quad \sqrt{\frac{c}{\omega L \cos \vartheta}}<\sin \vartheta \\
& \frac{\delta n}{n_{c}}>>\sin ^{2} \vartheta \sqrt{\frac{L}{l_{c x} \ln \left(\frac{L}{l_{c x}}\right)}} \mathrm{e}^{\frac{-K^{2} l_{c y}^{2}}{4}} ; \quad \frac{c}{\omega l_{c y}} \ll<\sin \vartheta
\end{aligned}
$$

These formulae are obtained by normalizing (20) and (21) with a coefficient $\sqrt{\frac{L}{l_{x} \ln \left(L / l_{x}\right)}}$ obtained from [[13]. Therefore the dependence of criteria on radial correlation langth is inherited from [[13] and explanation of physics behind this dependence is unclear.

While this procedure is based on assumption of the concidence of nonlinearity and saturation criteria in the case $\vartheta=0$, direct confirmation of which seems challenging, the numerical validation is possible. The result of full-wave modelling presented both in [10] and in latter sections of this paper demonstrate agreement with the generalized formulae (23) and (24).

The generalized expression (23) explicitly coincides with the one obtained in [10]. While the parameters determining transition to nonlinear regime include turbulence correlation length, that is not nesecessarily known in experiment, formulae (23) and (24) still provide some information about possibility of obtaining linear scattering regime in experiment.

\section{Analytical treatment discussion}

Having obtained criteria for transition to nonlinear regime, we would like to consider their relevance to experiment. But before doing that all the limitation of the model should be accounted for.

\subsection{Model limitations}

First and the most experimentally limiting approximation of the model is that of the radially uniform density fluctuations in the turning point area. It was used to obtain the solution of equation (4). To overcome this limitation, generalized formulae (23) and (24) are proposed, though they are not rigorously derived from basic equations.
Another simplification used in this model is linear dependency of $k^{2}$ on $x$, which allowed to compute explicitly integrals over $\mathrm{x}$ in (9) and (12). In experiment, the $k^{2}$ profile in the turning point vicinity, where Doppler backscattering takes place, can be approximated by a linear behavior. The corresponding $k^{2}$ profile scale length should replace parameter $L$ in the obtained criterea. In this form criterea (21), (22) are also applicable to the Xmode probing.

This treatment also makes the description of the probing of an important case of H-mode plasma possible. Such plasma features a strong density gradient at the edge followed by a flat area deeper in the plasma. While the gradient scale length inside the pedestal is different than at the edge, the edge area does not contribute strongly into scattered signal when cut-off is located in the flat area due to the reverse dependence on $k(x, K)$ of integrals over $x$ in formulae (9) and (12). Therefore analytical results are still relevant inside the pedestal when a gradient scale length corresponding to a flat area is used as $L$.

Another assumption that was used is (8), which generally holds true in the Doppler backscattering experiment.

The slab plasma geometry considered in the paper is consistent with experiments at the medium and large scale devices. The geometrical effects introduced by magnetic surface curvature are not leading to qualitative effects even on small device, as it was shown recently in [15].

Finally, we used WKB approximation, but the same results can be obtained by using exact Airy solutions, though derivation includes more complicated expressions. This was demonstrated in [15] for the Born approximation.

\subsection{Experimental relevance}

While formulae (23) and (24) provide criteria of nonlinear transition for a wide parameter range we would like to note the ones relevant to experimental parameters.

In FT-2 Doppler reflectometry experiment [17] the probing parameters for O-mode lead us to $L=1.5 \mathrm{~cm}$, $f=30 \mathrm{GHz}$. Radial and poloidal correlation lengthes determined from experimental data are 0.2-0.4 cm and are in agreement with gyrokinetic simulation results. In such a scenario, formula (24) is applicable and for the probing angle of $35^{\circ}$ used in experiment and the threshold value of $\delta n / n_{c}$ is roughly 0.2 , which is higher than the one required for saturation in this case. Therefore, our threshold (24) predicts that nonlinear regime can not be obtained under these experimental conditions, though curvature effects not included in the model might be significant in this case. 
Another example is ASDEX Upgrade Doppler reflectometry experiment [18] with simultaneous measurement of $l_{c y}$ and $l_{c x}$. In this case $L=10 \mathrm{~cm}$, $f \sim 30 \mathrm{GHz}$. The correlation lengthes defined from experiment are $l_{c y} \sim 1 \mathrm{~cm}$ and $l_{c x} \sim 3 \mathrm{~cm}$. Authors also propose corrected values $l_{c y}, l_{c x} \sim 0.6 \mathrm{~cm}$, which are based on numerical calculations performed to account for broadening of correlation in radial correlation reflectometry measurements predicted by linear theory [19] and similar effect in poloidal correlation reflectometry. For either pair or values, the relevant formula is (24) and normal probing used in experiment does not provide a possibility for nonlinear regime. In the case of probing under similar conditions, but with a tilted antenna, formula (24) would be the most relevant. Assuming that $l_{c y}$ and $l_{c x}$ value obtained in [18] are relevant to another L-mode ASDEX Upgrade Doppler reflectometry experiment, we can consider diagnostic setup with tilted antenna [20]. In this case for $f=60 \mathrm{GHz}$ corresponding $L$ is roughly $20 \mathrm{~cm}$ which means formula (24) is relevant again. For a probing angle $\vartheta=20^{\circ}$ formula (24) provides threshold value of $\delta n / n_{c}$ of 0.04 , which is significantly higher than the threshold $\delta n / n_{c} \sim 0.003$ provided by physical optics.

To conclude, formulae (24) seem to be the most relevant for experimental parameters, in particular (24c) describes the situation where it is possible to observe nonlinear scattering regime between linear and saturated regimes.

\subsection{Qualitative picture}

The term $\delta n(-2 K)$ in formula (10) corresponds to the face that the main contribution to the scattering signal in the linear (Born) approximation is provided by the fluctuation fulfilling the Bragg rule $q=-2 K$. In the next order of perturbation theory the Bragg rule should be also fulfilled which means that the total poloidal wavenumber of the fluctuations taking part in the scattering is also $2 K$, which is evident from formula (11). However, having multiple scattering events allows a certain degree of freedom in selecting the poloidal wavenumber of each separate fluctuation. The combination of the Gaussian spectra in formula (14) and (17) leads to $q=-K$ being the poloidal wavenumber providing dominant contribution to the scattered signal in the case of quadratic approximation. This is also represented by the exponents in formulae (23) and (24).

These arguments woud also apply for further orders of perturbation theory, which means that for $\mathrm{j}$-th order the dominant contribution to the scattering signal would be provided by fluctuations with $q=-2 K / j$. That in turn means, that scattered signal power would be proportional to $\exp \left(-K^{2} l_{c y}{ }^{2} / j\right) \times\left(\delta n / n_{c}\right)^{j}$.
Taking this into account, one can speculate, that with the growth of $\delta n$ consecutively higher orders would dominate the scattered signal, as they have weaker exponential supression. This speculation explains the results of numerical modelling presented further as well as numerical results of [10].

\section{Numerical calculations}

To validate obtained theoretical results, a full-wave numerical modelling with IPF-FD3D code [9] was performed. Two types of calculation were carried out.

In the first one, fluctuations of density uniform along radial direction were generated and applied to linear density profile uniform along the poloidal direction. This calculation is the closest to analytical model considered. An example of input density for FD3D computation can be seen on figure 2 .

Second type of calculations was performed for more realistic $2 \mathrm{D}$ turbulence applied to the same background profile.

Like analytical analysis, calculations were performed in the slab geometry. To generate random turbulence the density fluctuation Fourier transform amplitude $\delta n(\kappa, q)=\delta n_{0} \sqrt{l_{c y} l_{c x} / 8 \pi} \exp \left[-q^{2} l_{c y}^{2} / 8-\kappa^{2} l_{c x}^{2} / 8\right] \quad$ was used. Random phaseswere added to the amplitude of each harmonic and then Fourier transformation was performed. Each random turbulence field obtained this way was set to ha r.m.s. of 1 and then scaled to a certain fraction of $n_{c}$.

Each calculation was performed for 6000 separately generated turbulence fields. The calculation results were then averaged to produce the final result presented in this paper. This way a realistic random turbulence was used in calculation while it's spectral properties were still in agreement with analytical model.

All the calculations were performed for a "frozen" density without temporal dependence in agreement with analytical model.

An expamples of densities for two types of calculations can be seen on figures 2 and 3 respectively. For the first figure, $l_{c y}=1 \mathrm{~cm}$ and turbulence r.m.s. is set to $0.1 \mathrm{n}_{\mathrm{c}}$. Probing frequency $f$ is $40 \mathrm{GHz}$ and $L$ is $20 \mathrm{~cm}$. In the second figure radial correlation length of the turbulence is $1 \mathrm{~cm}$ and the rest of parameters are same as in figure 2.

An issue that was adressed during calculation was the fact that uniform turbulence shown in figures 2 and 3 displays a very high turbulence level at plasma edge. To exclude this effect the turbulence was suppresed at the edge by multiplying the initial tubtulent function to a hyperbolic tangent with a step located at $L / 2$. It should be noted, however, that in most cases, including the ones presented in this paper, this suppresion did not change the 
outcome of full-wave computation leading us to a conclusion that strong edge turbulence did not significantly influence the scattered signal in presented cases in the first place.

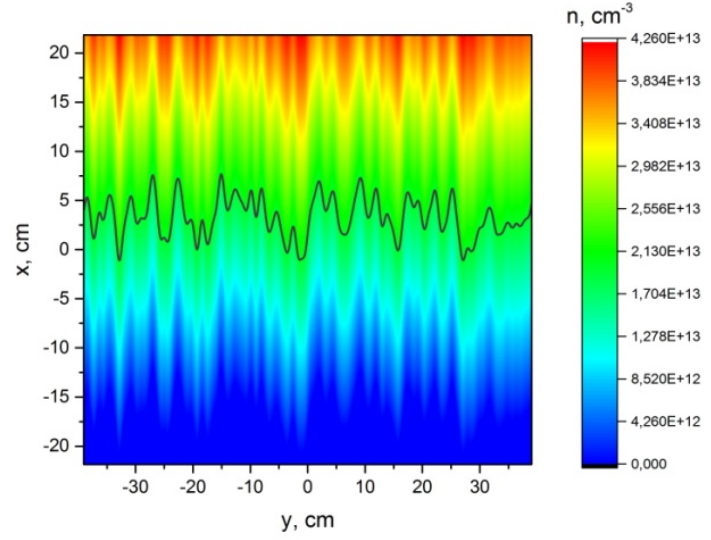

Figure 2. Density with random fluctuations uniform in the radial direction. Grey line designates cut-off density for the probing frequency used in FD3D calculations.

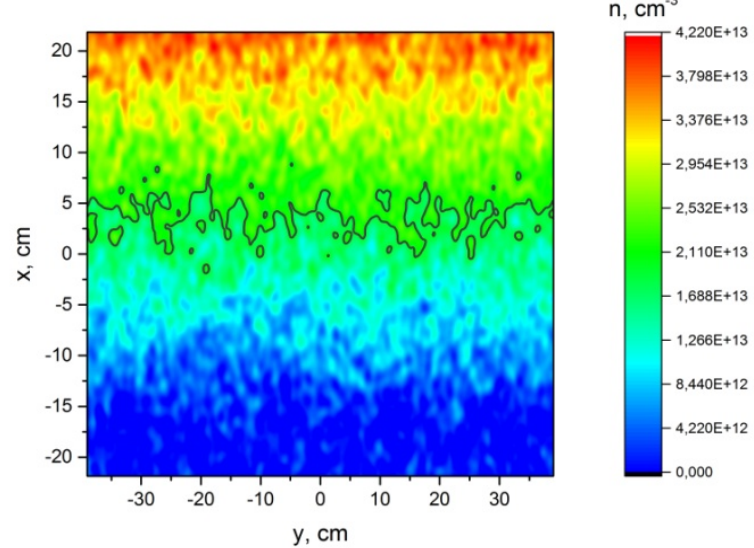

Figure 3. 2D density with random fluctuations. Grey line designates cut-off density for the probing frequency used in FD3D calculations.

\subsection{Modelling results for nonlinearity criterion}

For the calcualtion with radially homogenous turbulence $L=20 \mathrm{~cm}, \vartheta=40^{\circ}, l_{c y}=1.3 \mathrm{~cm}$ and $\rho=4 \mathrm{~cm}$ were used.

On the figure 4, scattered signal power depending on turbulence amplitude is plotted. Turbulence amplitude characterized by as follows: r.m.s. $(\delta n)=a \times n_{c}$. Lower half of the graph also shows the power scaling value $n_{i}=\ln \left(P_{i+1} / P_{i}\right) / \ln \left(a_{i+1} / a_{i}\right)$. The subscript $\mathrm{i}$ in this formula refers to a specific value of tubulence r.m.s. $a_{i}$ used in a calculation. The value $n_{i}$ is a characteristic of the power dependance of scattered field power with the growth of turbulnce amplitude: $P \propto a^{n}$

In the linear regime scattered field amplitude depends linearly on turbulence r.m.s. which means that dependence of scattered signal power is quadratic and the value of $n_{i}$ is 2 , which is indeed the case for small values of a. As the fluctuations become stronger, higher regime on nonlinearity can be seen with $n_{i}$ increasing to higher numbers. After that the transition to the strong phase modulation regime occurs which leads to saturation of the scatterred power, which can be seen in $n_{i}$ decrease.

Vertical lines on the picture correspond to different criteria derived previously. Solid green line corresponds to approptiate for this case (20), dashed purple line is saturation criterion (22) and dotted orange line corresponds to physical optics result which is described by (21). It can be seen that for these parameters the difference between our consideration and physical optics results is quite significant. It seems that in the considered case of radially uniform turbulence our criteria predicts transition to nonlinear regime more precisely than physical optics model.

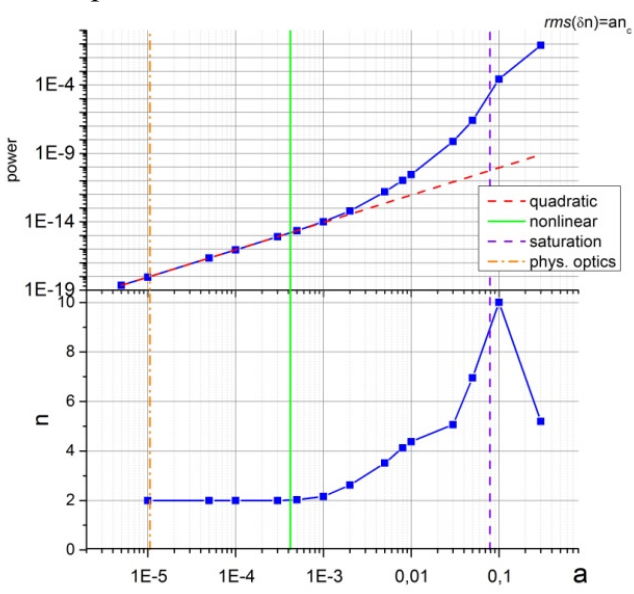

Figure 4. Scattered signal power calculated in case of radially uniform turbulence with $l_{c y}=1.3 \mathrm{~cm}$. The dashed red line corresponds to quadratic power dependence. Vertical lines correspond to nonlinearity thresholds provided by our analysis (green line), physical optics (orange dash-dotted line) and strong phase modulation condition (purple dashed line).

An example of a more realistic calculation with 2D turbulence is plotted on the figure 5. Probing beam parameters in this case were also $L=20 \mathrm{~cm}, \vartheta=40^{\circ}$, $l_{c x}=1.3 \mathrm{~cm}, l_{c y}=1.3 \mathrm{~cm}$ and $\rho=4 \mathrm{~cm}$.

The vertical lines in this case correspond to generalized version of the same criteria - solid green line is formula (24), the dash-dotted orange line is the criterion obtained in [10] and dashed purple line is saturation criterion $\frac{\delta n}{n_{c}}>>\frac{c}{\omega \sqrt{l_{c x} L \ln \left(L / l_{c x}\right)}}$. Just as in the case of $1 \mathrm{D}$ turbulence, in $2 \mathrm{D}$ calculations physical optics model underestimates the threshold density fluctuation amplitude for transition to nonlinear regime while our criterion provides a correct value. As can be seen from figure 5 , the difference between the two is more than of an order of magnitude which makes physical optics criterion significantly more limiting. 


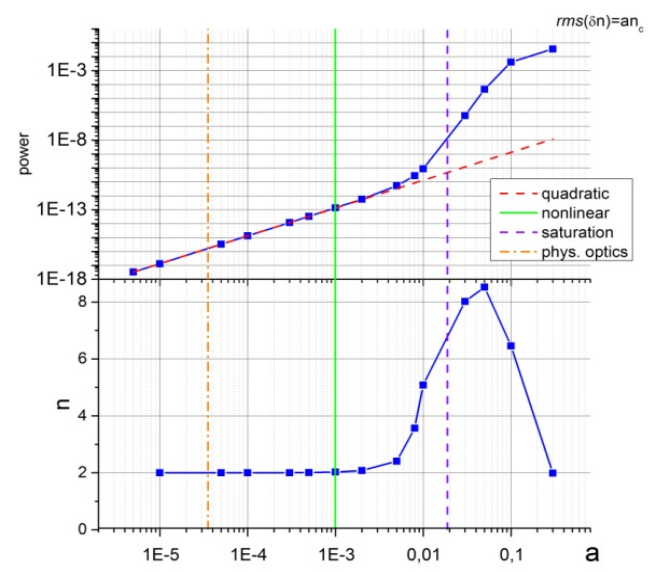

Figure 5. Scattered signal power and its exponential scaling in case of $2 \mathrm{~d}$ turbulence with $l_{c x}=l_{c y}=1.3 \mathrm{~cm}$. The dashed red line corresponds to quadratic power dependence. Vertical lines correspond to nonlinearity thresholds provided by our analysis (green line), physical optics (orange dash-dotted line) and strong phase modulation condition (purple dashed line).

However in the cases when (24) predicts nonlinear transition at density perturbation amplitudes so high that saturation would happen before nonlinear transition occurs, numerical results are not in agreement with (24). An example of such a calculation can be seen at figure 6 .

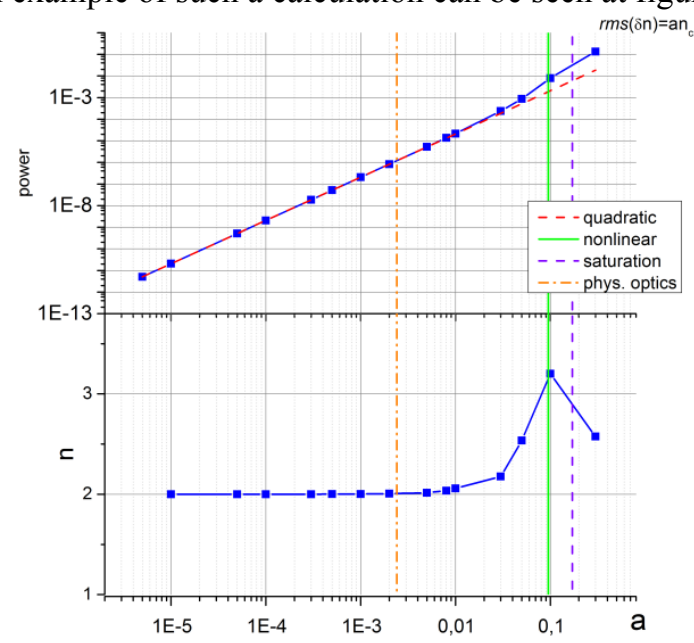

Figure 6. Scattered signal power calculated in case of radially uniform turbulence with $f=30 \mathrm{GHz}$ and $l_{c y}=0.6 \mathrm{~cm}$. The dashed red line corresponds to quadratic power dependence. Vertical lines correspond to nonlinearity thresholds provided by our analysis (green line), physical optics (orange dash-dotted line) and strong phase modulation condition (purple dashed line).

The possible cause of this discrepancy could be that in such situation $K l_{c y} \sim 1$, which is the limit of validity of formula (24). In this case formula (24) becomes relevant, however numerical coefficient associated with it is unclear since the integral estimation in that case was rather rough, which is why we neglected it. That omitted numerical coefficient is what we assume was the cause for discrepancy. However, an exact estimation of the coefficient and consequently validation of this explanation would require further study of analytical formulae derived in part 1 with numerical methods.

\subsection{Modeling result to validate qualitative picture}

To validate the explanation for a continuous growth of nonlinearity with the growth of $\delta n$ proposed in 2.3 a calculation with a step-like poloidal turbulence spectrum was performed:

$$
\delta n(\kappa, q)=\left\{\begin{array}{cc}
\delta n_{0} & |q|<k_{0},|\kappa|<k_{0} \\
0 & |q|>k_{0} \text { or/and }|\kappa|>k_{0}
\end{array}\right.
$$

The value of $k_{0}$ was selected so that $K<k_{0}<2 K$. For this spectrum according to our speculation, higher orders of nonlinearity would lose the exponential advantage provided by the Gaussian spectrum, while linear scattering would be suppressed due to absence of turbulence with high enough poloidal wavenumbers. The numerical values for the calculation were:

$f=30 \mathrm{GHz}, L=20 \mathrm{~cm}, \quad \vartheta=40^{\circ}$ (which means that $\left.K=4.4 \mathrm{~cm}^{-1}\right), \rho=4 \mathrm{~cm}$ and $k_{0}=5 \mathrm{~cm}^{-1}$. The result of the calculation is presented at figure 7 :

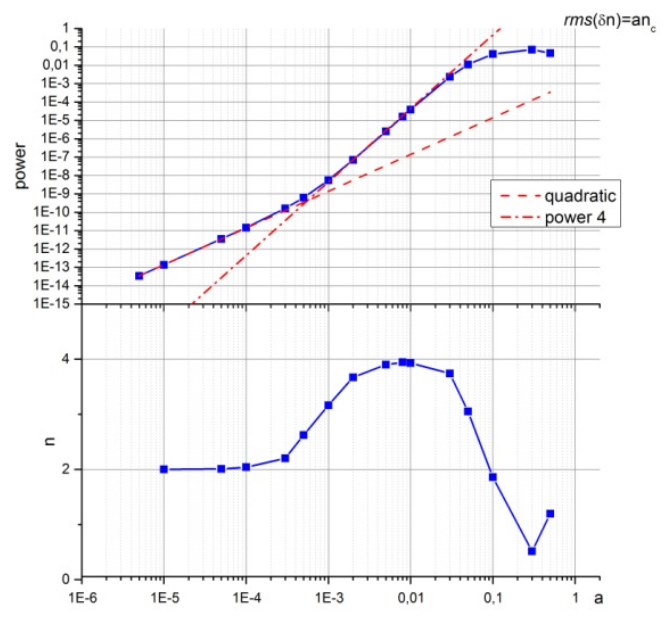

Figure 7. Scattered signal power and its exponential scaling in case of step-like spectrum. The dashed and dash-dotted red lines correspond to quadratic and quartic power dependencies respectively.

The value $n_{i}$ in this case does not exceed 4, which is in agreement with our expectations, as it means higher orders of scattering are no longer dominating scattered signal. This confirms our speculation that higher orders of scattering only become viable when fast decrease of turbulence amplitude with polodal wavenumber is present. At low turbulence amplitude linear scattering regime is observed despite the absence of fluctuations capable of producing linear scattering signal in the turbulence spectrum. This can be explained by the finite width of the probing beam which makes linear backscattering at lower poloidal wavenumbers possible. Such backscattering is suppressed by antenna diagram, but can dominate a scattered signal when turbulence amplitude is low enough.

\section{Conclusions}


In this paper we have analytically investigated Doppler reflectometry nonlinear scattering regime in the framework of perturbation theory. The analytical results were obtained for radially uniform plasma turbulence and then generalized to describe turbulence with arbitrary radial correlation length. Depending on the set up and plasma parameters different thresholds for a transition to nonlinear regime were established and compared to experimentvalues. Analytical results were validated with full-wave numerical modelling for both radially uniform and fully 2D turbulence and an agreement between analytical nonlinear thresholds and full-wave simulation results has been shown. This agreement shows that the thresholds obtained can be applied to experiments according to the mentioned restrictions. An explanation for scattered signal power dependance has been deduced from the extension to higher orders of perturbation method and validated numerically.

\section{Acknowledgements}

Financial support of RFBR grant 18-32-00031 is acknowledged by O.K., whereas support of RSF grant 17-12-01110 is acknowledged by E.G.

\section{Appendix A. Saturation criteria.}

A condition of strong phase modulation, which has been derived in [12], can be obtained for the assumption, used in this paper. Formula describing probing beam phase perturbation in [9] in our notation and under the assumption of 1D density fluctuations and linear background profile can be written as:

$$
\begin{aligned}
& \delta \varphi\left(k_{y}\right)=-\frac{\omega^{2}}{2 k_{y} c^{2}} \int_{0}^{y_{0}} \frac{\delta n(y)}{n_{c}} d y ; \\
& y_{0}\left(k_{y}\right)=4 k_{y} \frac{L c}{\omega} \cos \vartheta
\end{aligned}
$$

Then, the phase perturbation correlation function:

$$
\begin{aligned}
& \sigma_{j i}=\left\langle\delta \varphi\left(k_{y i}\right), \delta \varphi\left(k_{y j}\right)\right\rangle= \\
& =-\frac{\omega^{4}}{4 k_{y i} k_{y j} c^{4}} \int_{0}^{y_{0}\left(k_{y i}\right)} \int_{0}^{y_{0}\left(k_{y i}\right)} \frac{\left\langle\delta n(y) \delta n\left(y^{\prime}\right)\right\rangle}{n_{c}{ }^{2}} d y d y^{\prime} ;
\end{aligned}
$$

Assuming the turbulence to be statistically stationary and homogeneous:

$$
\sigma_{i i}=-\frac{\omega^{4}}{k_{y}{ }^{2} c^{4}} \int_{-\infty}^{\infty} \frac{|\delta n(q)|^{2}}{n_{c}{ }^{2}} \sin ^{2}\left(q y_{0}\left(k_{y i}\right) / 2\right) \frac{d q}{q^{2}} ;
$$

Now, assuming the turbulence has the Gaussian poloidal wavenumber spectrum and taking into account narrow antenna angular diagram, this integral can be estimated as:

$$
\sigma_{i i}= \begin{cases}\left(\frac{L \omega}{c} \cos \vartheta\right)^{2} \frac{\delta n^{2}}{n_{c}{ }^{2}} ; & \frac{L}{l_{c y}} \sin \theta \cos \vartheta<<1 \\ \frac{\omega^{2} l_{c y} L \cos \vartheta}{c^{2} \sin \vartheta} \frac{\delta n^{2}}{n_{c}{ }^{2}} ; & \frac{L}{l_{c y}} \sin \theta \cos \vartheta>>1\end{cases}
$$

Which leads us to formula (22). Here, once again, the numerical factors were neglected.

\section{References}

[1] Hennequin P et al. 2004 Rev. Sci. Instrum. 75, 3881 (2004)

[2] Conway G D et al 2005 Plasma Phys. Control. Fusion 47, 1165.

[3] Gusakov E Z and Surkov A V 2004 Plasma Phys. Control. Fusion 461143

[4] Bulanin V V Yafanov M V 2006 Plasma Phys. Rep. 3247

[5] Hirsch M, Holzhauer E, Baldzuhn J, Kurzan B and Scott B 2001 Plasma Phys. Control. Fusion 431641

[6] Gusakov E Z, Surkov A V, and Popov A Yu 2005 Plasma Phys. Control. Fusion 47959

[7] Heuraux S et al 2013 J. Phys.: Conf. Ser. 416012019

[8] Krutkin O L, Altukhov A B, Gurchenko A D et al. 2017 Proc. 44th EPS Conf. on Contr. Fusion and Plasma Physics (Belfast) ECA vol. 41F P2.108

[9] Lechte C et al 2017 Plasma Phys Control. Fusion 5907500

[10]Pinzon J R et al 2017 Plasma Phys Control. Fusion 59035005

[11]Piliya A D and Popov A Yu 2002 Plasma Phys Control. Fusion 44467

[12]Gusakov E Z and Popov A Yu 2004 Plasma Phys Control. Fusion 461393

[13]Gusakov E Z and Popov A Yu 2002 Plasma Phys Control. Fusion 442327

[14]Beckmann P and Spizzichina A 1963 The Scattering of Electromagnetic Waves from Rough Surfaces (Oxford: Pergamon)

[15]Gusakov E Z and Krutkin O L 2017 Plasma Phys. Rep. 43, 605-613.

[16]Gusakov E Z and Tyntarev M A 1997 Fusion Eng. Des. 34-35 501

[17]Altukhov A B et al 2016 Plasma Phys. Control. Fusion 58 105004

[18]Prisiazhniuk D et al 2018 Plasma Phys. Control. Fusion 60 075003

[19]Gusakov E Z and Yakovlev B O 2002 Plasma Phys. Control. Fusion $\mathbf{4 4} 2525$

[20]Conway G D et al 2004 Plasma Phys. Control. Fusion 46951 